Internationaler Herbert Falk Award 2017

\title{
Preis für Mikrobiomforscher
}

Für seine außergewöhnlichen Leistungen auf dem Gebiet der Gastroenterologie, wurde Prof. Joseph Sung, Präsident der Chinesischen Universität Hongkong, in Berlin mit dem „Internationalen Herbert Falk Award 2017 "ausgezeichnet.

Das Forschungsgebiet von Sung sind die chronisch entzündlichen Darmerkrankungen (CED), insesondere die Rolle des Mikrobioms beim Morbus Crohn und der Colitis ulcerosa. Für die mittlerweile auch in Asien steigende CED-Inzidenz macht Sung vor allem eine mit dem veränderten Lebensstil assoziierte Änderung des Mikrobioms verantwortlich, wobei dem „ländlichen Mikrobiom“ aus seiner Sicht eine protektive Rolle zukommen könnte. Sung und sein Team gehen derzeit vor allem der Frage nach, welche Bedeutung die Kolonisation des Darms mit Bakterien in der frühen Kindheit hat, welche Faktoren konkret für die Veränderung des Mikrobioms verantwortlich

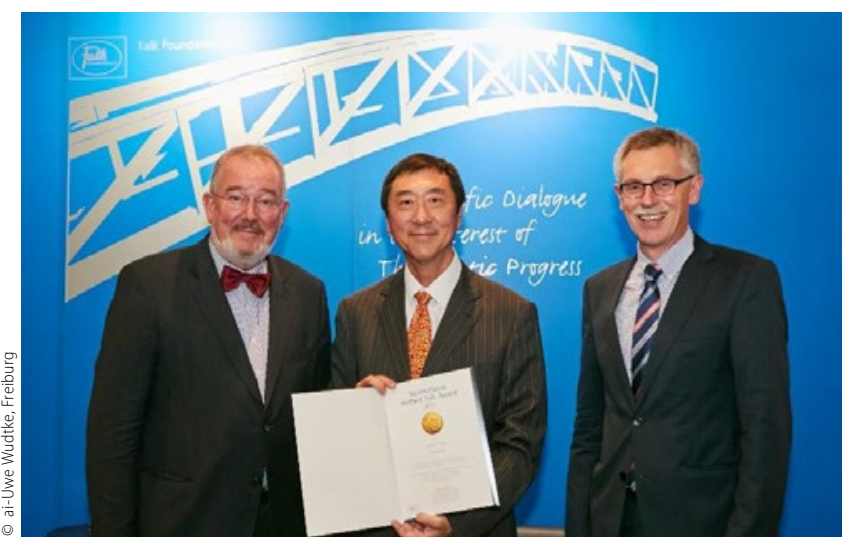

v.l.n.r.: Prof. Jürgen Schölmerich (Preiskomitee), Prof. Joseph Sung (Preisträger), Dr. Roland Greinwald ( für die Falk Foundation)

sind, wie sie sich auf die Krankheitsentwicklung der CED auswirken und wie das Mikrobiom ein metabolischen Syndroms triggern kann. Sie gehen dabei auch der Frage nach, wie diätetische Maßnahmen eine mikrobielle Selektion bedingen und welche Bedeutung poten- zielle Interaktionen des Mikrobioms mit Viren, Pilzen oder mit einer Wurmbesiedlung des Darmes haben können.

Nach Information von Falk zum Symposium 209 "IBD 2017 - Therapeutic and Biological Barriers", Berlin, 6. - 7.10.2017, Falk Foundation e.V.

\section{Mit Fixkombination Behandlungslücke geschlossen}

Mit dem Single-Tablet-Regime (STR) Sofosbuvir/Velpatasvir/Voxilaprevir können nun auch bei Hepatitis-C-Patienten aller Genotypen, die bereits erfolglos mit direkt antiviral wirkenden Substanzen (DAA) behandelt wurden, mit einer zwölfwöchigen Therapiedauer Heilungsraten von 96-98\% erzielt werden.

\section{Gute Heilungsoption}

Seit gut einem Jahr stehe mit der Fixkombination $400 \mathrm{mg}$ Sofosbuvir/100 mg Velpatasvir (SOF/VEL, Epclusa ${ }^{\circledR}$ ) das damals erste pangenotypische DAA-Regime zur Verfügung, mit dem je nach Stadium der Lebererkrankung oder Vortherapie über eine zwölfwöchige Therapiedauer mit und ohne Ribavirin bei mindestens $95 \%$ der Patienten eine Heilung der chronischen Hepatitis C erzielt werde (Fachinformation Epclusa ${ }^{\oplus}$ Stand: Mai 2017), erinnerte Prof. Jörg Petersen, Leitender Arzt Innere Medizin am ifi-Institut für interdisziplinäre Medizin Hamburg. Das seit Juli 2017 zugelassene STR mit 400 mg Sofosbuvir/100 mg Velpatasvir/100 mg Voxilaprevir (SOF/VEL/VOX, Vosevi ${ }^{\circledR}$ ) biete nicht nur therapienaiven Patienten ohne Zirrhose (acht Wochen Therapiedauer) oder mit kompensierter Zirrhose (zwölf Wochen Therapiedauer), sondern auch erstmals DAA-vorbehandelten $\mathrm{Pa}$ tienten eine gute Heilungsoption (Fachinformation Voseviं; Stand. Juli 2017), betonte Petersen. In den Studien mit DAA-vorbehandelten Patienten erzielte SOF/VEL/VOX Heilungsraten von 96-98\% [Bourliere $M$ et al. N Engl J Med 2017; 376: $2134-46]$.

\section{Eliminierung der Hepatitis C rückt in greifbare Nähe}

Mit den bestehenden Heilungschancen im Praxisalltag rücke die Eliminierung von Hepatitis C in greifbare Nähe, konstatierte Petersen. Es sei dafür aber not- wendig, die vermutlich hohe Dunkelziffer an bisher nicht diagnostizierten HCVInfizierten zu senken und mehr Patienten als bisher zu behandeln.

\section{Wann ist kostenloses Screening möglich?}

Für Petersen kommt dabei den Hausärzten eine Schlüsselrolle als primärer Ansprechpartner für Personen mit einem erhöhten HCV-Risiko zu. Auch ohne Screeningprogramm sei ein kostenloses Screening mit drei Fragen in der klinischen Praxis möglich: Könne eine der Fragen wie zu Migrationshintergrund, intravenöser Drogenkonsum/Gefängnisaufenthalt/homosexueller Mann, Bluttransfusion vor 1992, bejaht werden, bestehe ein berechtigter Verdacht auf eine Hepatitis $\mathrm{C}$ und ein budgetneutrales Testen sei mit der Laborziffer 32006 machbar.

Andrea Warpakowski

Pressekonferenz „Auf dem Weg zur Hepatitis-CEliminierung - Heilung jetzt auch für schwer zu behandelnde Patienten mit einer DAA-Vorbehandlung möglich“ am 14.9.2017, Dresden, im Rahmen des DGVS-Kongresses; Veranstalter: Gilead Sciences 